Case Report

\title{
An Unusual Presentation of Scimitar Syndrome in a Military Service Member
}

\author{
Daniel B. Simmons, ${ }^{1}$ Ravi S. Menon, ${ }^{1}$ William L. Pomeroy, ${ }^{1}$ \\ Travis C. Batts, ${ }^{1}$ and Ahmad M. Slim ${ }^{1,2}$ \\ ${ }^{1}$ Cardiology Service, Brooke Army Medical Center, San Antonio, TX 78234, USA \\ ${ }^{2}$ Cardiology Service, San Antonio Army Medical Center, MCHE-MDC, 3851 Roger Brooke Drive, San Antonio, TX 78234-6200, USA
}

Correspondence should be addressed to Ahmad M. Slim; ahmad.m.slim.mil@mail.mil

Received 20 May 2013; Accepted 27 June 2013

Academic Editors: C.-L. Hung and C. Pizzi

Copyright ( 2013 Daniel B. Simmons et al. This is an open access article distributed under the Creative Commons Attribution License, which permits unrestricted use, distribution, and reproduction in any medium, provided the original work is properly cited.

\begin{abstract}
This is the case of a twenty-two-year-old active duty male soldier with nonexertional chest pain and worsening performance on his physical fitness test. His history was significant for a diagnosis of dextrocardia upon entry to the military. On acute presentation to the emergency department, he was deemed a candidate for the expedited coronary computed tomographic angiography (CCTA) protocol to assess for a possible anatomic cause of his symptoms. CCTA revealed the presence of an anomalous right pulmonary vein draining into the inferior vena cava. Additionally, the imaging showed dextroversion of the heart, dilation of the inferior vena cava, right atrium, and right ventricle, as well as a hypoplastic right lung, a collection of findings consistent with scimitar syndrome and not dextrocardia.
\end{abstract}

\section{Case Report}

This is a case report of a twenty-two-year-old male soldier with nonexertional chest pain and worsening performance on his physical fitness test. His history was significant for a diagnosis of dextrocardia upon entry to the military. On physical examination, heart sounds were louder over the right hemithorax; there was a fixed split S2, and the point of maximal impulse was displaced to the right. He had normal lung sounds and no peripheral edema. An electrocardiogram was remarkable for left axis deviation of the $p$-wave, normal QRS axis, and a nonspecific intraventricular conduction delay.

Because of his history of recurrent chest pain and vague history of dextrocardia not confirmed with ECG, patient underwent a cardiac computed tomographic angiography (CCTA) protocol to assess for a possible anatomic cause of his symptoms. CCTA, performed with sequential gating and a window of $40-80 \%$ to accommodate the large area under investigation, revealed the presence of an anomalous right pulmonary vein draining into the inferior vena cava. Additionally, the imaging showed dextroversion of the heart, dilation of the inferior vena cava, right atrium, and right ventricle, as well as a hypoplastic right lung, a collection of findings consistent with scimitar syndrome and not dextrocardia (Figure 1). There was no evidence of pulmonary sequestration or an atrial septal defect.

Review of the patient's chest radiograph showed a classic scimitar sign, which is the presence of a scimitar-shaped pulmonary vein along the right cardiac border (Figure 2). Due to concerns about RV enlargement, cardiac magnetic resonance imaging was performed and confirmed the finding of right ventricular enlargement on CCTA and estimated the right ventricular ejection fraction at 35\% (Figure 3). Catheterization of the right heart revealed a step-up in oxygenation of the upper inferior vena cava consistent with a left-to-right shunt, normal right-sided pressures without evidence of pulmonary hypertension, and a pulmonary to systemic shunt fraction (Qp/Qs) of 1.63.

\section{Discussion}

Scimitar syndrome is a rare form of anomalous pulmonary venous return with an estimated incidence of $2 / 100,000$ in 


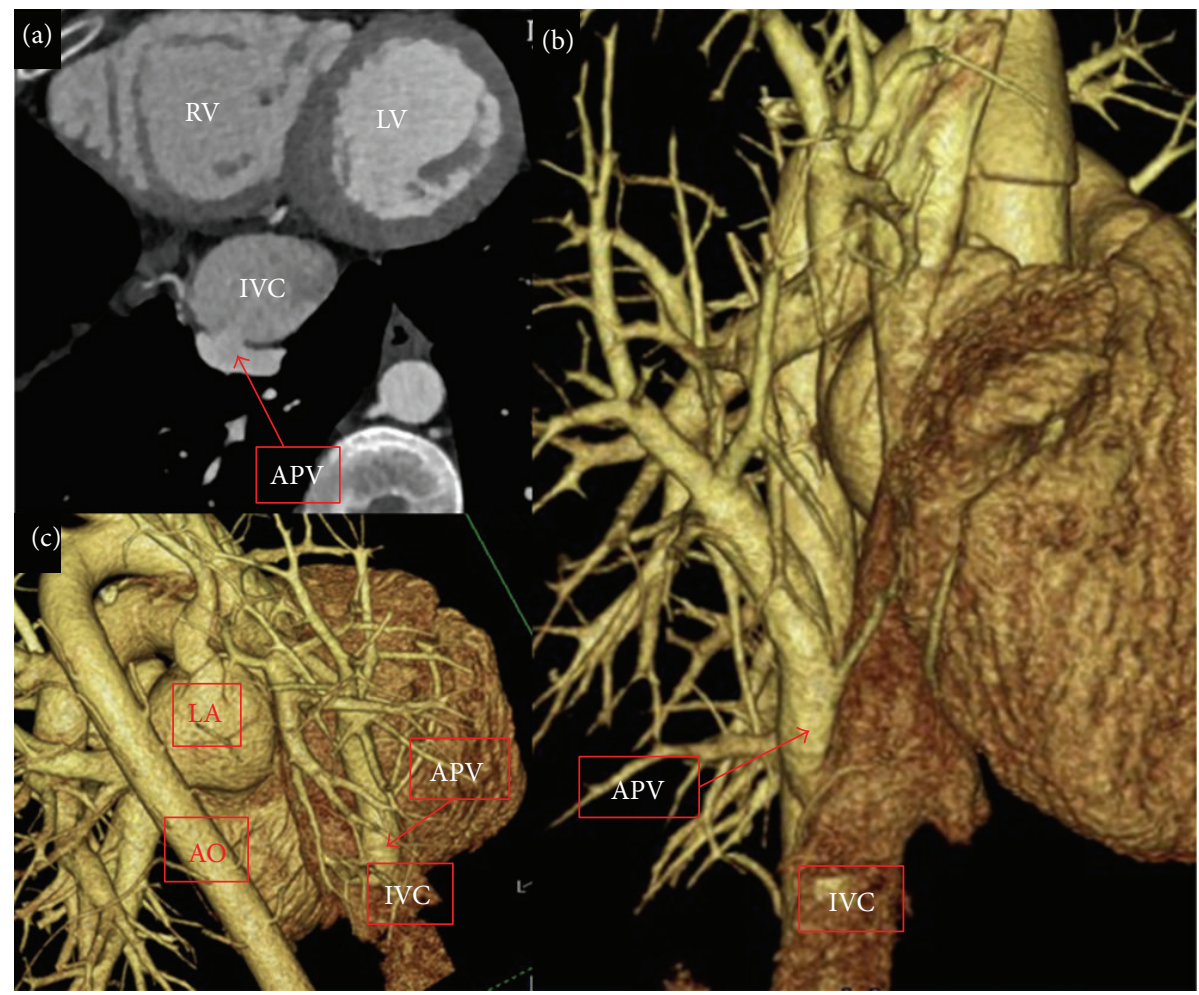

FIGURE 1: Axial and coronal images with sequential gating; cardiac phases utilized of $40-80 \%$ on CCTA $(\mathrm{kVp}=120$ with care dose variable $\mathrm{mAs}$ and slice thickness of $0.7 \mathrm{~mm}$ with 0.4 overlap). They revealed the presence of an anomalous pulmonary venous connection between the right lower lobe pulmonary vein (APV) and the enlarged inferior vena cava (IVC) [a, b, and c].

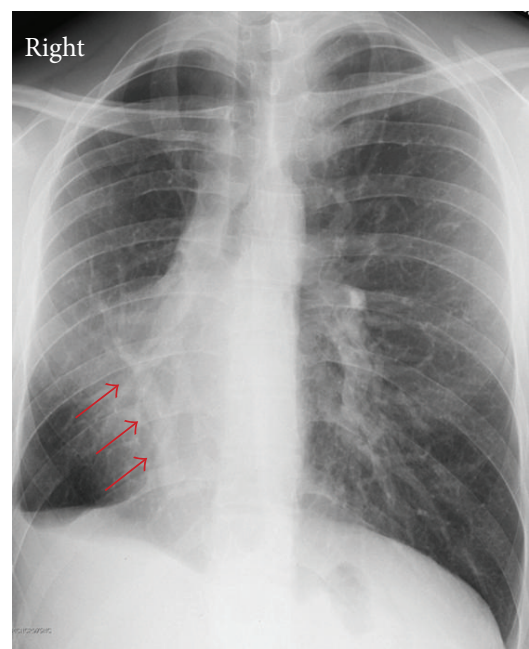

Figure 2: Chest radiograph shows decreased space associated with the hypoplastic lung and displacement of the cardiac silhouette to the right (scimitar sign) (arrows).

which all or part of the venous drainage from the right lung enters the inferior vena cava instead of the left atrium [1]. The syndrome derives its name from the classical appearance of the chest radiograph, in which the shape of the anomalous pulmonary vein resembles the curved Turkish sword [2]. The severity of symptoms depends on the degree

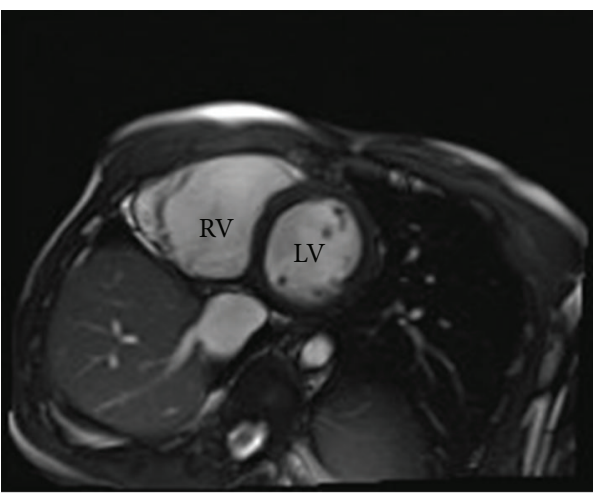

FIgURE 3: Cardiac magnetic resonance imaging showing right ventricular (RV) enlargement as compared to the left ventricular (LV) size with calculated right ventricular ejection fraction of $35 \%$.

of the shunt present and may be manifested as dyspnea on exertion, palpitations, syncope, or congestive heart failure [3]. Pulmonary sequestration with systemic arterial supply and atrial septal defect is present in approximately 50\% and 20\% of adults, respectively [1]. There are three variants of scimitar syndrome: an infantile form which is diagnosed before one year and is associated with heart failure and pulmonary hypertension, an adult form with milder symptoms, and a form associated with other congenital heart malformations [4]. 
Scimitar syndrome has classically been diagnosed in the cardiac catheterization laboratory, but CCTA-as part of a routine screening protocol in our institution for lowrisk chest pain-was essential to make the diagnosis. It also demonstrated the right-sided pulmonary hypoplasia and dextroversion of the heart which are the most common abnormalities associated with scimitar syndrome. The patient ultimately proceeded to surgical correction of the anomalous pulmonary venous return, and CCTA was vital in operative planning and successful surgical repair.

\section{Conclusion}

Scimitar syndrome is a rare variant of anomalous pulmonary venous return that is usually diagnosed in infanc, but may present in adults with symptoms of dyspnea on exertion, syncope, or heart failure. In this twenty-two-year-old soldier with prior combat deployments, CCTA was vital in the diagnosis of scimitar syndrome. This vascular anomaly and the associated congenital malformations likely led to the soldier's decreased exercise tolerance and were successfully repaired by a congenital heart specialist.

\section{Disclaimer}

The opinions or assertions contained herein are the private views of the authors and are not to be construed as reflecting the Department of the Army or the Department of Defense.

\section{References}

[1] U. Gudjonsson and J. W. Brown, "Scimitar syndrome," Pediatric Cardiac Surgery Annual, vol. 9, no. 1, pp. 56-62, 2006.

[2] L. Midyat, E. Demir, M. AşkIn et al., "Eponym: scimitar syndrome," European Journal of Pediatrics, vol. 169, no. 10, pp. 1171-1177, 2010.

[3] A. A. Korkmaz, C. E. Yildiz, B. Onan, M. Guden, G. Cetin, and K. Babaoglu, "Scimitar syndrome: a complex form of anomalous pulmonary venous return," Journal of Cardiac Surgery, vol. 26, no. 5, pp. 529-534, 2011.

[4] C. Dupuis, L. A. C. Charaf, G.-M. Breviere, P. Abou, M. RemyJardin, and G. Helmius, "The 'adult' form of the scimitar syndrome," American Journal of Cardiology, vol. 70, no. 4, pp. 502-507, 1992. 


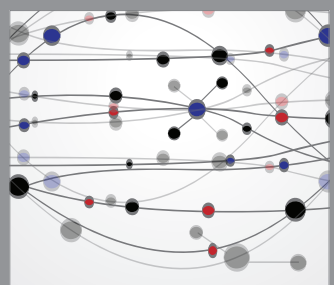

The Scientific World Journal
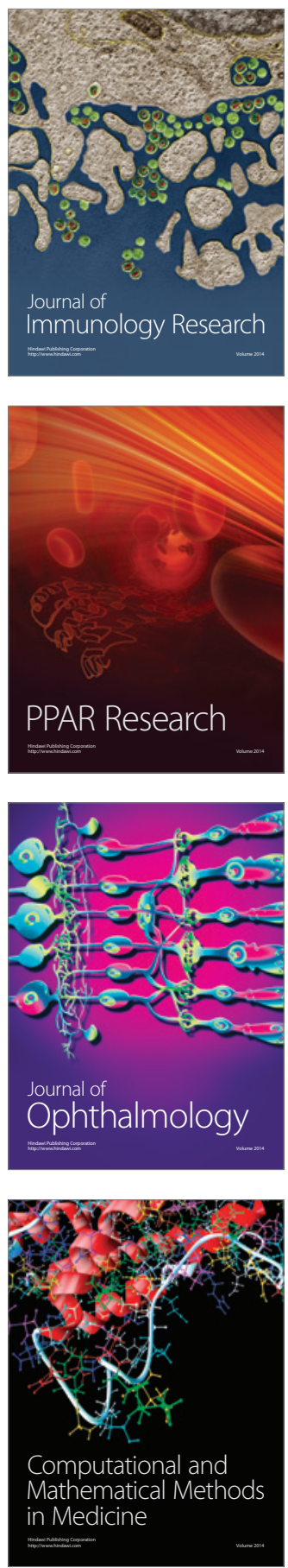

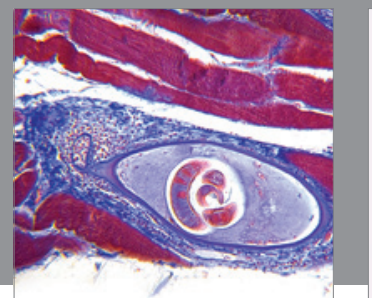

Gastroenterology

Research and Practice
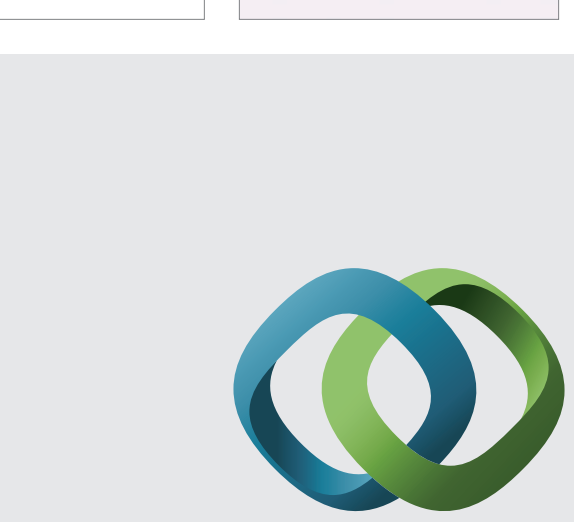

\section{Hindawi}

Submit your manuscripts at

http://www.hindawi.com
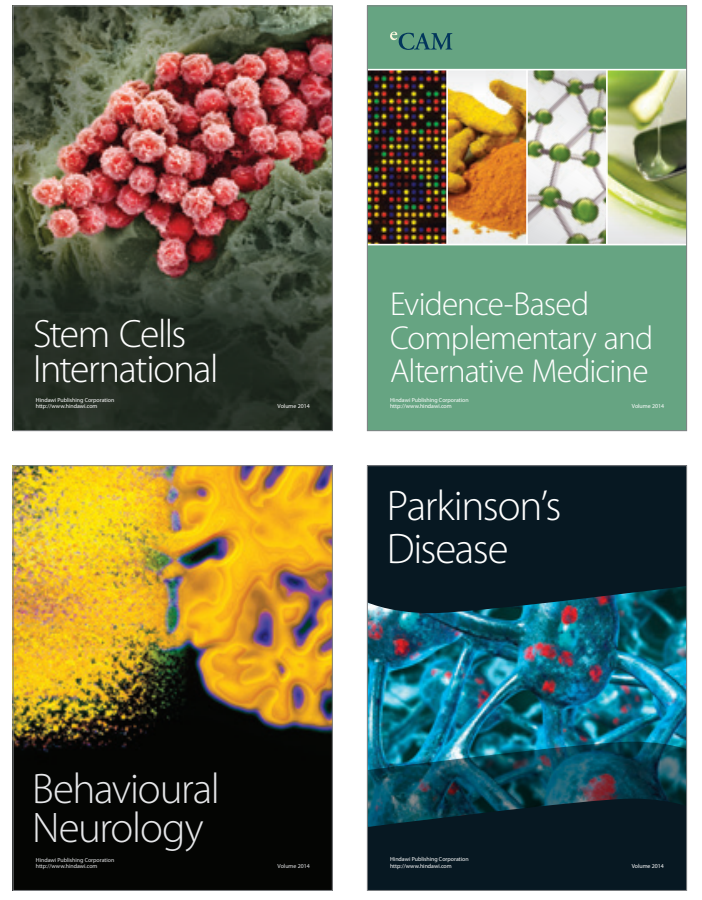
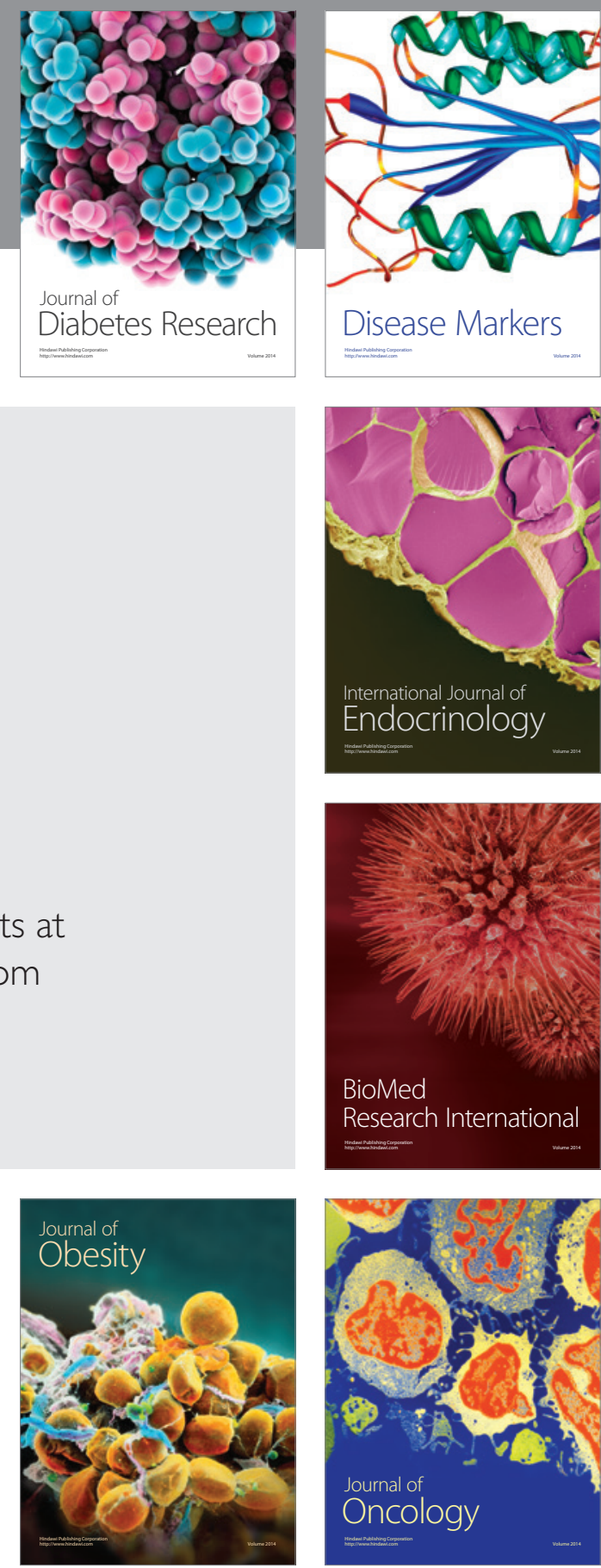

Disease Markers
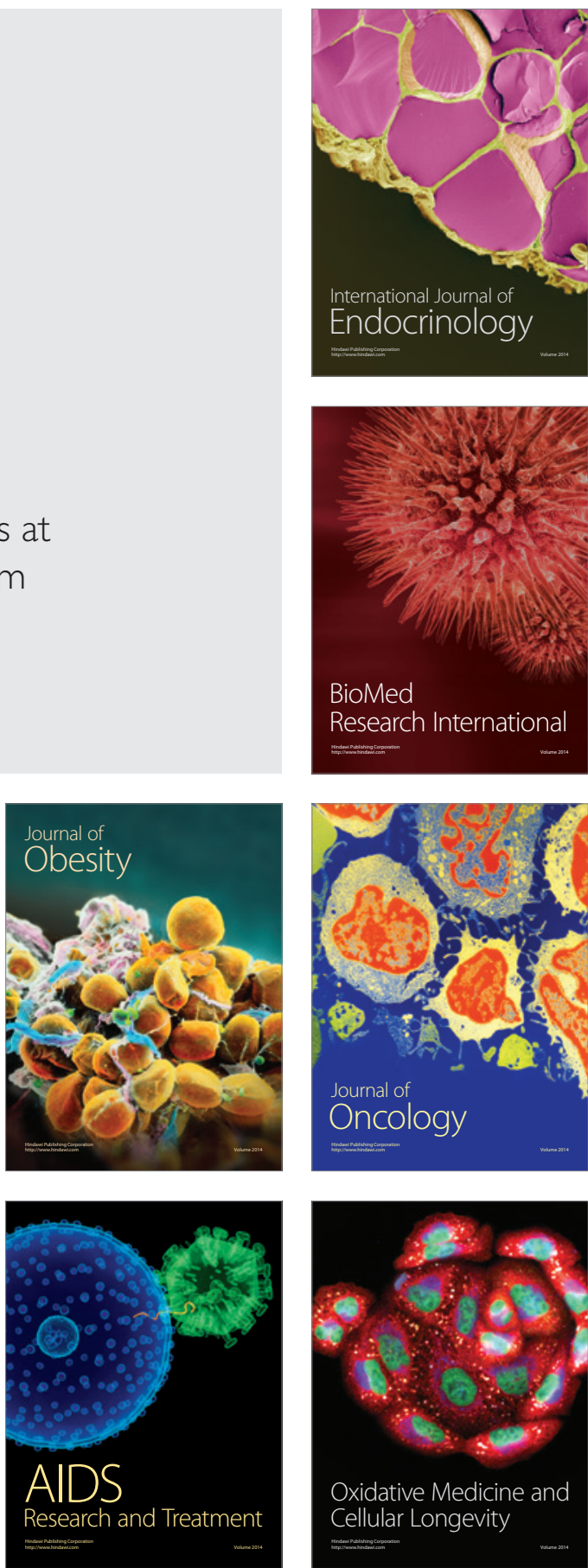